\title{
Random amplitude fatigue life of electroformed nickel micro-channel heat exchanger coupons
}

\author{
Larry Byrd*, Michael P. Camden, Gene E. Maddux \\ and Larry W. Simmons \\ Structures Division, Air Vehicles Directorate, \\ Air Force Research Laboratory, 2790 D Street, \\ Building 65, Wright-Patterson AFB, \\ OH 45433-7542, USA
}

Received 24 February 1997

Revised 1 December 1997

The use of micro-channel heat exchangers (MCHEX) with coolant flow passage diameters less than $1 \mathrm{~mm}$ has been proposed for heat flux, weight, or volume limited environments. This paper presents room temperature, random amplitude, $\varepsilon-N$ (strain versus number of cycles to failure) curves for MCHEX coupons formed by electroplating nickel on a suitable form. These coupons are unique in two aspects; the microstructure formed by electroplating and the presence of holes as an integral part of the structure. The hole diameters range from approximately $10 \%$ to $50 \%$ to the specimen thickness. The fatigue life of electroformed nickel can be estimated from constant amplitude data using the formulation presented. The heat exchangers with channels parallel to the coupon direction have a lower fatigue life than the solid material.

\section{Introduction}

The use of micro-channel heat exchangers (MCHEX) which have coolant flow passage diameters less than $1 \mathrm{~mm}$ has been proposed for a number of applications. These include high heat flux, weight or volume limited environments such as computer microprocessors or the leading edges of hypersonic vehicles. A variety of manufacturing methods have been used to produce the heat exchangers. Most methods join a face sheet to

\footnotetext{
${ }^{*}$ Corresponding author. Tel.: +1 937255 5059; E-mail: larry. byrd@flight.wpafb.af.mil.
}

a grooved back sheet by brazing or diffusion bonding, and then braze manifolds to the heat exchangers. The heat exchangers to be studied in this paper were constructed by electroplating nickel on a suitable form to create a thin sheet with holes in the plane of the sheet. The final thickness was obtained by grinding. Two geometry's were available; round or roughly rectangular channels as shown in Fig. 1.

The objective of the study was to create a room temperature $\varepsilon-N$ curve for the MCHEX when exposed to narrow band random excitation around the first fundamental bending mode. The coupons are unique in two aspects: the microstructure formed by electroplating and the presence of holes as an integral part of the structure. One question to be answered was; could standard fatigue data for nickel be used to predict the fatigue life of the MCHEX. Since the outer fiber of the material is the most highly stressed, the channels may not be significant. On the other hand, channels, especially rectangular ones, may present stress concentrations that result in failure modes that differ from a solid specimen. The results imply that this may happen in the MCHEX.

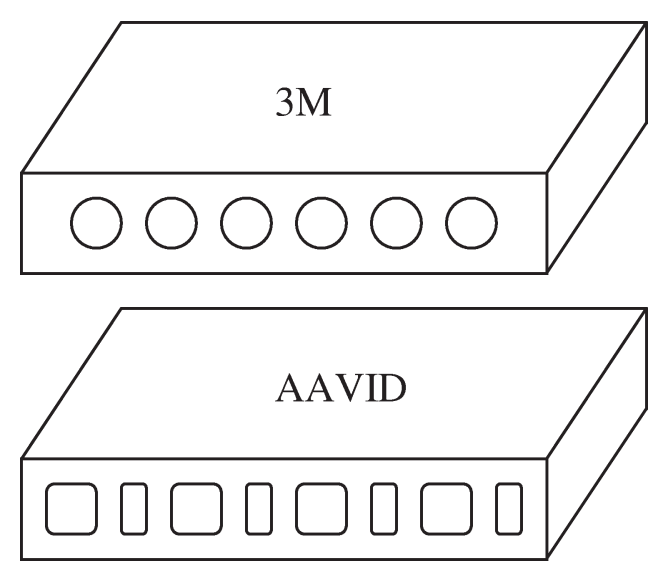

Fig. 1. MCHEX with holes perpendicular to the long axis of the coupon. 
Not all the specimens were tested in time to be included in this paper. The available coupons came from two sources, 3M Co. and AAVID Laboratories, but were all manufactured at $3 \mathrm{M}$. The specimens were meant to prove the manufacturing concept rather than for production, so no specific quality control procedures were followed. It may be possible to improve the quality by studying the failed specimens to eliminate the worst failure modes if they can be related to the manufacturing process. The nickel that results from the electroforming process is in a soft state similar to an annealed condition. It is possible to get a harder material by the addition of the proper additives during the plating. The specimens to be discussed are either solid or have channels parallel to the long axis of the coupon and were produced by $3 \mathrm{M}$.

\section{Test specimens}

The specimens were all nominally $25.4 \mathrm{~mm}$ wide $\times$ $102 \mathrm{~mm}$ long $\left(1^{\prime \prime} \times 4^{\prime \prime}\right)$ except one solid specimen which was $127 \mathrm{~mm}\left(5^{\prime \prime}\right)$ long. The $3 \mathrm{M}$ MCHEX were $1.09 \mathrm{~mm}\left(0.043^{\prime \prime}\right)$ thick and have round holes with a diameter of $0.508 \mathrm{~mm}\left(0.02^{\prime \prime}\right)$. The orientation of the channels was parallel to the long axis of the coupons. The solid specimens were $1.04 \mathrm{~mm}\left(0.042^{\prime \prime}\right)$ thick. These specimens were received ground on both sides.

The single AAVID specimen tested was $1.32 \mathrm{~mm}$ $\left(0.052^{\prime \prime}\right)$ thick with channels that were alternately wide and narrow. The narrow channels were nominally $0.0498 \mathrm{~mm} \times 0.162 \mathrm{~mm}\left(0.002^{\prime \prime} \times 0.0064^{\prime \prime}\right)$ while the wide ones were nominally $0.0747 \mathrm{~mm} \times 0.162 \mathrm{~mm}$ $\left(0.003^{\prime \prime} \times 0.0064^{\prime \prime}\right)$. As seen in Fig. 2 these channels were not perfectly uniform in shape. The orientation of the channels was perpendicular to the long axis of the coupon. As received the AAVID specimens varied

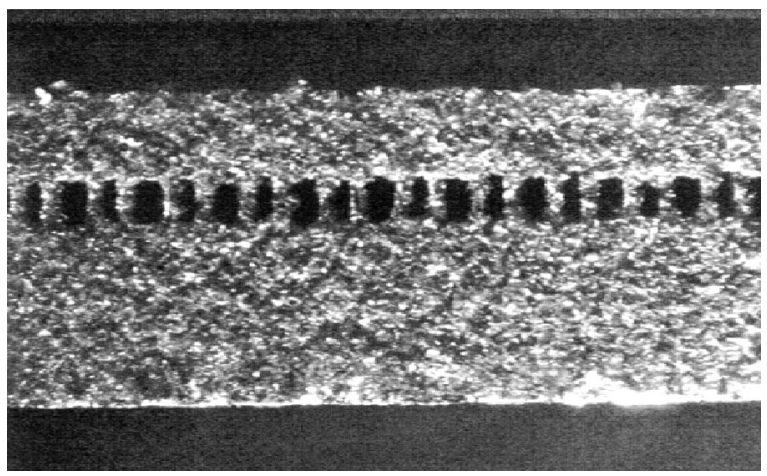

Fig. 2. Photomicrograph of AAVID specimen. significantly in thickness so they were ground on the side farthest from the holes to achieve uniform thickness but this was not enough to place the holes at the center of the coupon. The surface finish on the ground side was $0.8 \mu \mathrm{m}$ and the other was in the as-received condition. Due to manufacturing tolerances the channel centers did not form a perfect line along the center of the cross section for either heat exchanger type.

\section{Instrumentation and test setup}

The shaker tests were conducted at the Wright Laboratory in the Structural Dynamics branch. The specimens were cantilevered one at a time on a shaker. The first $25.4 \mathrm{~mm}\left(1^{\prime \prime}\right)$ of the coupon was in the fixture for a free length of $76.2 \mathrm{~mm}\left(3^{\prime \prime}\right)$. The only exception was one of the solid specimens which was $127 \mathrm{~mm}\left(5^{\prime \prime}\right)$ long with a free length of $102 \mathrm{~mm}\left(4^{\prime \prime}\right)$. Measurements Group, Inc WK-07-125AD-350 strain gages were attached to both sides of the specimen at the root to provide strain data. Due to the fact that strain gages often fail during high cycle fatigue testing, a laser vibrometer was also used to monitor strain. A vibrometer measures velocity at a given point on a test specimen, this velocity is then integrated into displacement. The displacement is then plotted versus strain from the strain gages. Strain versus displacement points are plotted for multiple input levels (all below the test level). Because this relationship is linear, and independent of temperature, strain can be computed from this plot using only the integrated velocity from the vibrometer. The specimens were excited with a low level broad band signal to determine the first bending mode and then excited with a narrow band random signal (bandwidth $\approx$ $100 \mathrm{~Hz}$ ) centered around the fundamental frequency. The shaker input was set to achieve a desired RMS strain and the data acquisition system recorded the fundamental frequency and the number of accumulated cycles. The system is shown schematically in Fig. 3. More details can be found in Camden et al. [1]. The tests were run until a significant decrease in natural frequency indicated failure. This is typically $10 \%$ for metals, but will vary with the material being tested. For the nickel specimens tested in this study, a $2 \%$ shift in the resonance was used to define failure. The failure criteria is covered in more detail in the discussion of results. 
SHAKER TEST ARRANGEMENT FOR RANDOM RESPONSE TESTS

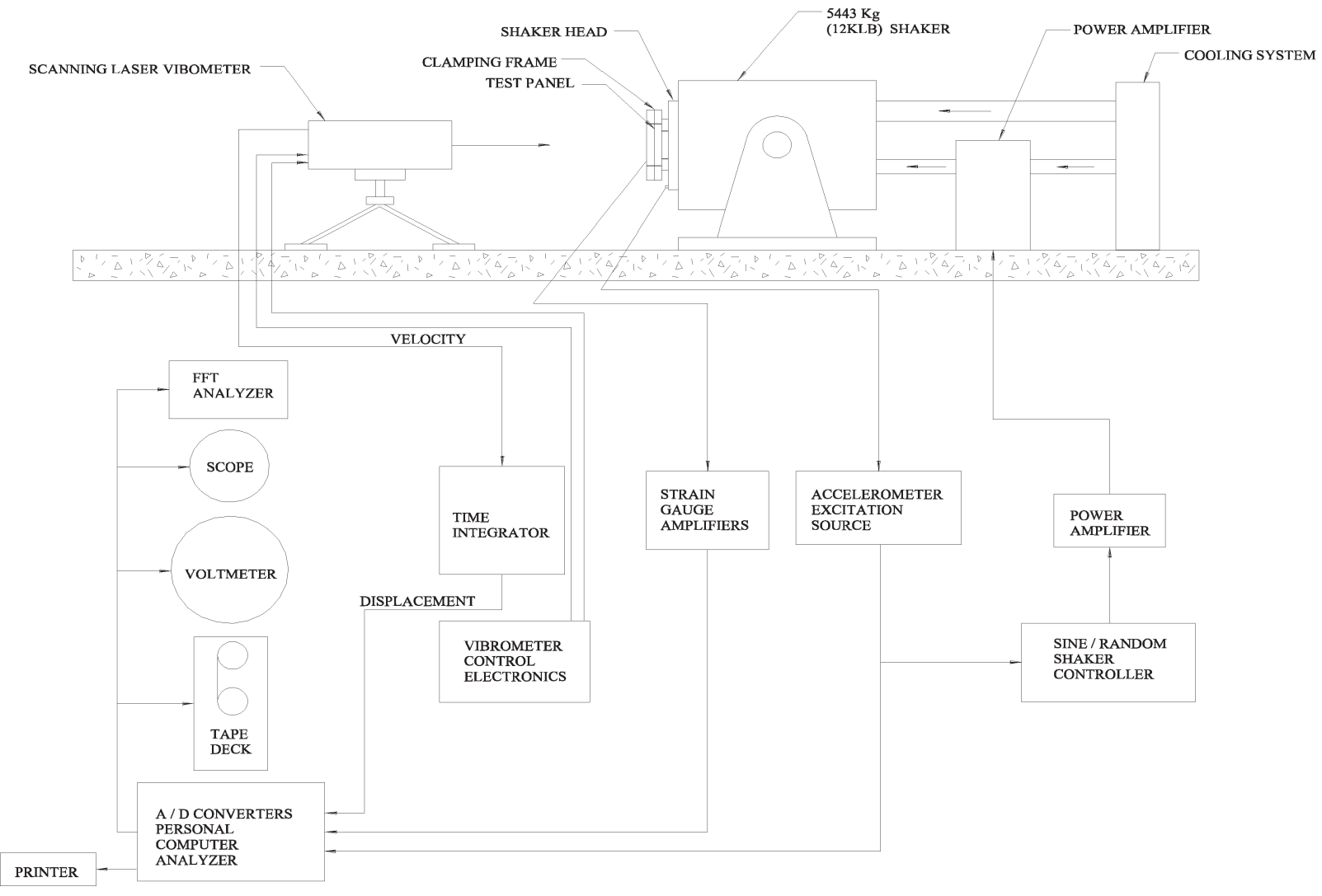

Fig. 3. Test set up.

\section{Theoretical considerations}

It is desirable to compare the experimental results with those found for conventionally formed nickel specimens to assess the manufacturing process. The authors were not able to find narrow band random results so a conversion from data taken using sine excitation was used. A conservative method suggested by Rudder and Plumbee [6] uses Miner's cumulative damage rule and assumes the random excitation follows a Rayleigh distribution.

It is given by:

$$
N_{\mathrm{r}}(\sigma)=\left[\int_{0}^{\infty} \frac{p(s)}{N_{\mathrm{s}}(s)} \mathrm{d} s\right]^{-1},
$$

where $N_{\mathrm{r}}(s)$ is the number of cycles to failure in a random excitation environment described by an RMS stress $s ; N_{\mathrm{s}}(s)$ is the number of cycles to failure in a constant amplitude sine excitation at stress level $s$. The Rayleigh distribution is given as:
Table 1

Curve fit constants for nickel data of the form $N(s)=a s^{b}$

\begin{tabular}{lcc}
\hline Sine data, cut direction & $a(\mathrm{ksi})^{-b}$ & $b$ \\
\hline parallel & $4.15901 \times 10^{-21}$ & -10.1317 \\
perpendicular & $1.76042 \times 10^{-23}$ & -12.1507 \\
\hline
\end{tabular}

$$
p(s)=\frac{s}{\sigma^{2}} \mathrm{e}^{-s^{2} /\left(2 \sigma^{2}\right)} .
$$

The upper limit of integration was set at $496 \mathrm{MPa}$ (72000 psi) and $N(s)$ was calculated for two different cuts of nickel sheet subjected to fully reversed bending with zero mean stress. One cut formed specimens parallel to the direction the sheet was rolled and the other perpendicular. Published data from Grover et al. [2] was curve fit in a least squares fashion to a power law with the form $N(s)=a s^{b}$ and the integral calculated numerically. Table 1 gives the values of $a$ and $b$ for the different curve fits. This was then converted to strain as a function of cycle count to be compatible with the experimental data. 


\section{Strain vs Cycles to Failure ( $2 \%$ Decrease in Fundamental Frequency)}

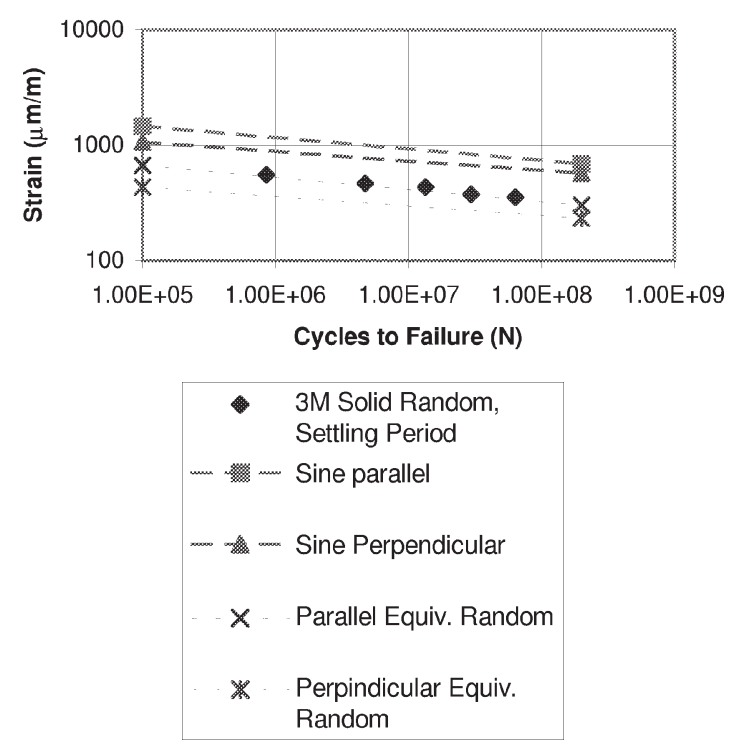

Fig. 4. Comparison with published data.

\section{Results}

Figure 4 compares the experimental results for the solid material with the published sine data and equivalent random curves. The top two lines are the published constant amplitude curves which are typically much higher than random data. The bottom two lines are the equivalent random excitation curves calculated from Eq. (1). The symbols are the solid nickel data and can be seen to be slightly higher but in reasonable agreement with the parallel random equivalent curve. The data points were calculated using a settling period as will be discussed later in more detail.

Figure 5 presents the MCHEX data. The dashed curves are the same equivalent random curves as in Fig. 4. The single AAVID data point is seen to lie roughly on the same line as the solid material. More data will be needed to draw any conclusions about that material. The results for the 3M MCHEX are shown as two different sets of data corresponding to two different methods of handling the data. The first method does not account for a settling period for any of the runs. The second method attempts to separate an initial period where the natural frequency drops rapidly before leveling off. Since it may be questioned that this is appropriate, both results were included. It can be seen that the corrected data is bracketed by the equivalent random curves with a similar slope. The uncorrected data

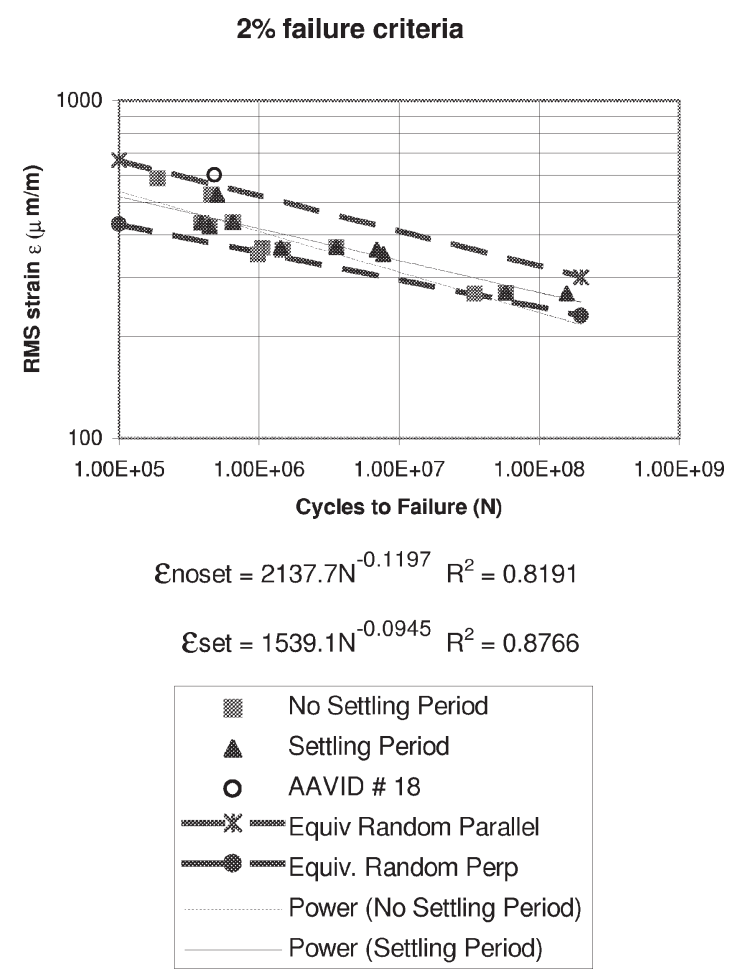

Fig. 5. MCHEX $\varepsilon-N$ curve for channels parallel to the coupon axis.

has a larger slope and thus is more conservative. However, it also lies between the random equivalent curves for $N$ less than approximately $5 \times 10^{7}$. The MCHEX data has significantly more scatter than the solid material regardless of the method chosen for calculating the failure point. Using the corrected data does reduce scatter. This can be seen from the values for $R^{2}$ for the curve fits shown in Table 2. The closer the $R^{2}$ value is to one, the better the curve fits the data. The maximum deviation from the line and the standard deviation of the deviation from the line is also shown in Table 2 . The corrected data shows two values for $R^{2}$ and the maximum deviation. The smaller values are arrived at by not using the value for $3 \mathrm{M}$ specimen \#2 because it was determined that it could be a spurious data point using Chauvenet's criteria as given by Holman. In the uncorrected data this point also showed a large deviation from the line but not large enough to delete from the data set.

The deviation from the curve fit was calculated as:

$$
\begin{aligned}
& \% \text { deviation } \\
& =\frac{(\text { Exp. Value })-(\text { Curve Fit Value })}{\text { Curve Fit Value }} \times 100 \text {. }
\end{aligned}
$$


Table 2

Data curve fit (strain $\left.=a N^{b}\right)$ statistics

\begin{tabular}{lccccc}
\hline Specimens & Settling period & No. of points & Curve fit $R^{2}$ & Max. dev. \% & Stand. dev. \\
\hline 3M Solid & No & 5 & 0.1731 & 22.3 & 15.4 \\
3M Solid & Yes & 5 & 0.9855 & 3.4 & 2.1 \\
MCHEX & No & 11 & 0.8191 & 17.6 & 10.5 \\
MCHEX all data & Yes & 11 & 0.8447 & 20.2 & 9.9 \\
MCHEX without \# 2 & Yes & 10 & 0.8766 & 18.3 & 7.8 \\
\hline
\end{tabular}

The standard deviation is calculated from the deviations in the usual manner. The data for the solid material without using a settling period was distorted significantly by one data point because there were only five specimens. Other than that point the data was similar for both methods. As can be seen the maximum and standard deviations are very good for the solid material when the settling period was used. For the heat exchangers the standard deviation is smaller when the settling period is used although the maximum percent error is larger.

The modulus of elasticity for the solid material was calculated using the standard equation for vibration of a cantilever beam given as:

$$
\omega_{1}=\frac{11}{L^{2}} \sqrt{\frac{E I}{\rho A}},
$$

where $\omega$ is the first fundamental frequency (Hertz), $L$ is the free length of the specimen (in), $E$ is the modulus of elasticity (psi), $I$ is the second moment of area with respect to the centroidal axis $\left(\mathrm{in}^{4}\right), \rho$ is the density $\mathrm{lb}_{\mathrm{m}} / \mathrm{in}^{3}, A$ is the cross sectional area of the coupon $\left(\mathrm{in}^{2}\right)$. The elastic modulus was found to be roughly $1.39 \mathrm{GPa}\left(20.2 \times 10^{6} \mathrm{psi}\right) \pm 12.7 \%$ which is similar to cast nickel but considerably lower than $2.07 \mathrm{GPa}$ $\left(30 \times 10^{6} \mathrm{psi}\right)$ which is common for other forms of the material. These results have not been confirmed by static tests at this time.

\section{Discussion of results}

The discussion can be separated into two parts referring to the variability of the failure of the heat exchangers and the failure criteria used. Figure 6a shows a view of an aluminum specimen after failure. It displays the typical crack front bands associated with fatigue failure. Figure $6 \mathrm{~b}$ shows several nickel coupons after failure. They were deliberately broken completely after the test to reveal the crack plane. As can be seen,

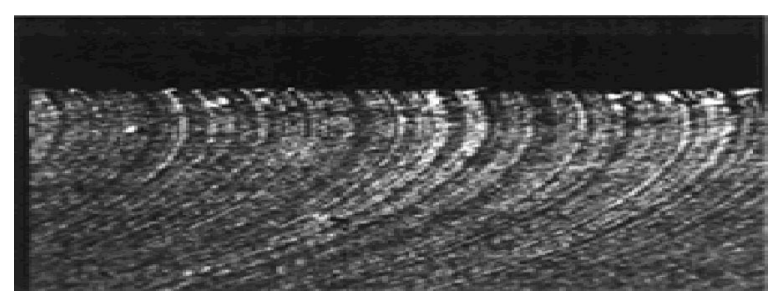

Fig. 6a. Typical bending fatigue failure in an aluminum specimen.

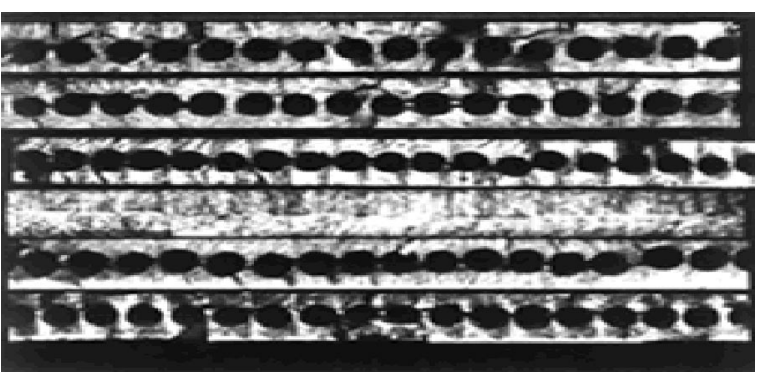

Fig. 6b. Typical bending fatigue failures of the nickel specimens tested.

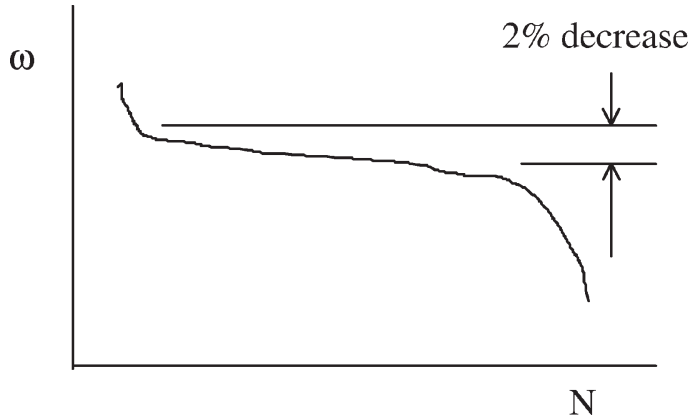

Fig. 7. Typical $\omega-N$ curve with a defined settling period.

even the solid specimen does not show the expected fracture surface. There are different failure modes occurring that may be due to a combination of property variations in the solid and because the channel did not all lie on the centerline. There appears to be a marked difference in the material properties in a rectangular area bounding each hole. Fracture surfaces emanate 
Resonant Frequency vs Cycle Count for AAVID \#18 perpendicular

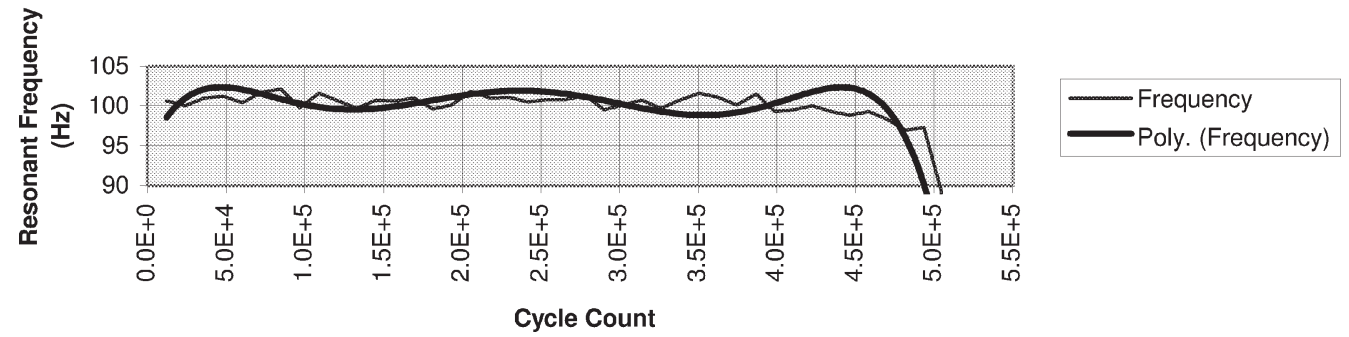

Resonant Freq vs No. Cycles 3M Solid \# 5

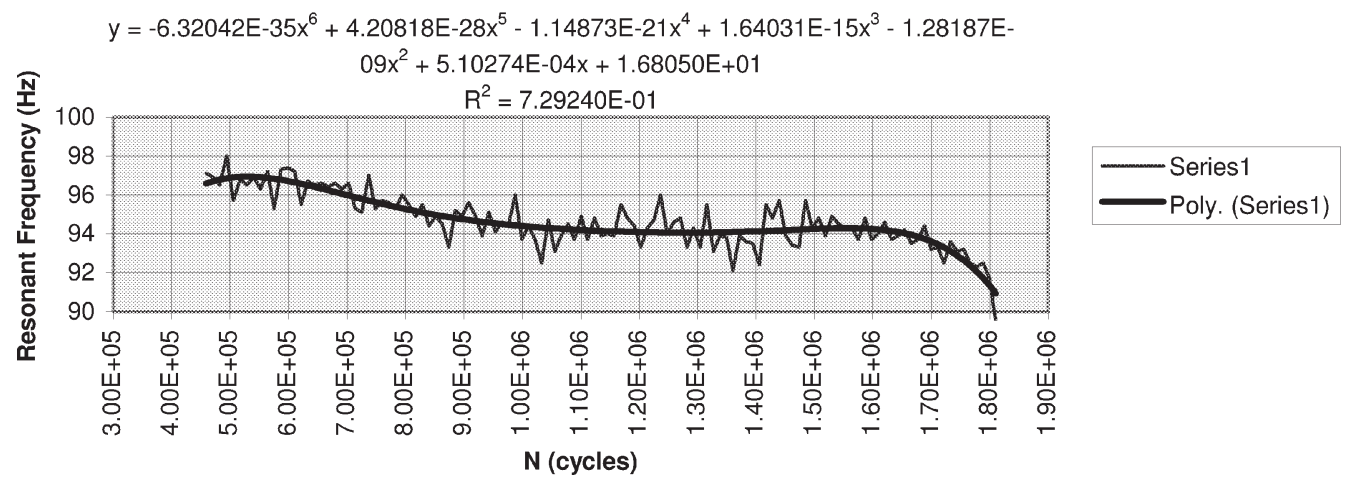

Fig. 8. Example of data smoothing with 6th order polynomial.

around each hole but stop at the boundaries between holes. Since some of the channels are closer to one surface, the bending stresses on the top and bottom vary, which could lead to the wide variations in fatigue life. Shear stresses which are a maximum along the neutral axis may also contribute to failure causing cracking between the holes and leading to a different failure mode.

The failure criteria was assumed to be a $2 \%$ decrease in the resonant frequency, $\omega$. This has been used by previous authors (Konig [4], Muller and Grunewald [5]) by examining the natural frequencycycle count curve to find a place where the curve becomes level after an initial "settling period" as shown in Fig. 7. This settling period is not always observed with metals but in this case the material was not isotropic and some of the specimens exhibited this behavior. The decrease in $\omega, \Delta \omega$, is measured from this initial natural frequency, $\omega_{0}$. Unfortunately, it is not always easy to tell where to choose $\omega_{0}$ due to scatter in the data or because there is no well defined leveling of the curve. The data was smoothed by fitting with a 6th order polynomial. In cases where a settling period could not be distinguished the value from the curve at the first recorded cycle count was used for $\omega_{0}$. It is still not certain that this is the best ap- proach because it inconsistently treats the data. That is why both results are shown in Fig. 5. The curve fit was also used to find the value for $N$ where $\Delta \omega$ was $2 \%$ of $\omega_{0}$. Figure 8 show examples of the fitted data. In some cases the curve fit drops before the experimental data. This may not cause that much of a problem if the fit is still adequate at the point where it is decided that failure has occurred. In other cases the curve fit has oscillations that do not seem to track the data as well as may be desired. Notice that the scatter is often larger than the $2 \%$ being used as the failure criterion so some type of smoothing is essential.

Table 3 shows the cycle count using different values for $\Delta \omega$. For much of the data there is not a large difference in the results if $2 \%, 3 \%$ or $5 \%$ is used for the failure criterion because $\omega$ drops quickly once it starts the sudden drop that signals complete failure of the specimen. It should be noted that the scatter will decrease if a larger value of $\Delta \omega$ is used but the results will be less conservative.

In the future it may be helpful to correlate the actual failure mechanism with the $\omega-N$ curve. This would give a better understanding of how to estimate remaining life from the decrease in natural frequency. Consider a simplistic approach using Eq. (4). This equation 
Table 3

Cycle count using different values of $\Delta \omega$ for failure criteria

\begin{tabular}{cccccc}
\hline Specimen & RMS $\varepsilon$ & $2 \%$ & $3 \%$ & $3.50 \%$ & $5 \%$ \\
\hline solid 5 & 550 & $8.57 \times 10^{5}$ & $1.66 \times 10^{6}$ & $1.71 \times 10^{6}$ & $1.78 \times 10^{6}$ \\
solid 6 & 430 & $1.25 \times 10^{7}$ & $1.31 \times 10^{7}$ & $1.33 \times 10^{7}$ & $1.37 \times 10^{7}$ \\
solid 6a & 463 & $4.76 \times 10^{6}$ & $4.83 \times 10^{6}$ & $4.86 \times 10^{6}$ & $4.93 \times 10^{6}$ \\
solid 7 & 373 & $2.98 \times 10^{7}$ & $3.04 \times 10^{7}$ & $3.06 \times 10^{7}$ & $3.12 \times 10^{7}$ \\
solid 8 & 353 & $6.39 \times 10^{7}$ & $6.52 \times 10^{7}$ & $6.56 \times 10^{7}$ & $6.69 \times 10^{7}$ \\
1 & 350 & $7.75 \times 10^{6}$ & $7.87 \times 10^{6}$ & $7.91 \times 10^{6}$ & $8.03 \times 10^{6}$ \\
2 & 587 & $1.89 \times 10^{5}$ & $2.87 \times 10^{5}$ & $2.96 \times 10^{5}$ & $3.12 \times 10^{5}$ \\
3 & 435 & $3.89 \times 10^{5}$ & $4.66 \times 10^{5}$ & $5.02 \times 10^{5}$ & $6.12 \times 10^{5}$ \\
4 & 437 & $6.52 \times 10^{5}$ & $7.05 \times 10^{5}$ & $7.23 \times 10^{5}$ & $7.65 \times 10^{5}$ \\
6 & 269 & $5.77 \times 10^{7}$ & $5.87 \times 10^{7}$ & $5.90 \times 10^{7}$ & $6.00 \times 10^{7}$ \\
7 & 424 & $4.41 \times 10^{5}$ & $4.94 \times 10^{5}$ & $5.17 \times 10^{5}$ & $5.79 \times 10^{5}$ \\
8 & 361 & $1.47 \times 10^{6}$ & $6.71 \times 10^{6}$ & $6.87 \times 10^{6}$ & $7.12 \times 10^{6}$ \\
9 & 527 & $4.58 \times 10^{5}$ & $5.03 \times 10^{5}$ & $5.15 \times 10^{5}$ & $5.37 \times 10^{5}$ \\
10 & 366 & $1.05 \times 10^{6}$ & $1.30 \times 10^{6}$ & $1.41 \times 10^{6}$ & $1.71 \times 10^{6}$ \\
11 & 368 & $3.56 \times 10^{6}$ & $3.65 \times 10^{6}$ & $3.69 \times 10^{6}$ & $3.77 \times 10^{6}$ \\
12 & 268 & $1.57 \times 10^{8}$ & $1.60 \times 10^{8}$ & $1.61 \times 10^{8}$ & $1.64 \times 10^{8}$ \\
\hline
\end{tabular}

implies that for the natural frequency to decrease, $E$ and/or $I$ must change. For most materials only moment of inertia, $I$, will change with the onset of cracking. This implies changes in the width and/or the thickness. For a uniform cross-section beam the change of inertia with accumulated loadings cannot be measured. Referring again to Fig. 6a, even though the position of the crack tip at the surface can be recorded as a function of accumulated cycles, the location of the leading edge of the crack at any point below the surface cannot be determined. The crack front curves and becomes parallel to the midline where the bending stresses are zero. A similar crack (not shown in the figure) will approach the midline from the bottom of the specimen. Complicated specimens such as those in Fig. $6 \mathrm{~b}$ do not show any recognizable evidence of crack growth and do not provide any basis for estimating fatigue damage from frequency degradation. Is $2 \%$ change a better number to use than $3 \%$ ? It is more conservative but when the $\omega-N$ curve is flat, as seen in some of the data, the error in the number of cycles can be very large. The important question seems to be how far away from total failure is this? This seems to be a problem in fracture mechanics especially when there is more than one failure mode. A careful study would require some means of inspecting the specimen at specified intervals or possibly doing residual strength tests on a large number of coupons. Until a means of accounting more precisely for the actual change in inertia, we can only gather an accumulation of test data and look for trends.

\section{Conclusions}

The fatigue life of electroformed nickel can be estimated from constant amplitude data using the formulation presented. The heat exchangers with channels with diameters approximately $50 \%$ of the coupon thickness and parallel to the coupon direction have a lower fatigue life than the solid material. They also show a large scatter, which seems to indicate different failure modes are occurring. However, the data below $5 \times 10^{7}$ cycles can still be bracketed by estimates from previously published data. It is expected that the fatigue life of MCHEX with channels running perpendicular to the coupon will have a lower fatigue life than one with parallel holes, but testing will be needed to confirm this. There is still not a good way to define failure. The method that gives the least scatter still involves visual examination of data and judgment of how it changes. This can lead to variation in interpretation which is still not resolved.

\section{References}

[1] M.P. Camden, L.W. Simmons and M.P. Banford, High temperature high cycle fatigue testing of carbon/carbon tee's, Wright Laboratory Technical Memorandum WL-TM-94-3051, 1994.

[2] H.J. Grover, S.A. Gordon and L.R. Jackson, Fatigue of Metals and Structures, US Government Printing Office, Washington, DC, 1954. 
[3] J.P. Holman, Experimental Methods for Engineers, 3rd edn, McGraw-Hill, 1978, p. 65.

[4] K. Konig, Acoustic fatigue testing on different materials and skin stringer elements, in: AGARD Conference Proceedings 549 Impact of Acoustic Loads on Aircraft Structures, Canada Communications Group, Quebec, 1994, pp. (13-1)-(13-11).
[5] G. Muller and M. Grunewald, Acoustic fatigue of carbon fibre structures, AGARD Conference Proceedings 549 Impact of Acoustic Loads on Aircraft Structures, Canada Communications Group, Quebec, 1994, pp. (15-1)-(15-7).

[6] F.F. Rudder and H.E. Plumbee, Sonic fatigue design guide for military aircraft, AFFDL-TR-74-112, AD-B004-600L, Wright-Patterson AFB, OH, May 1975, pp. 475-479. 

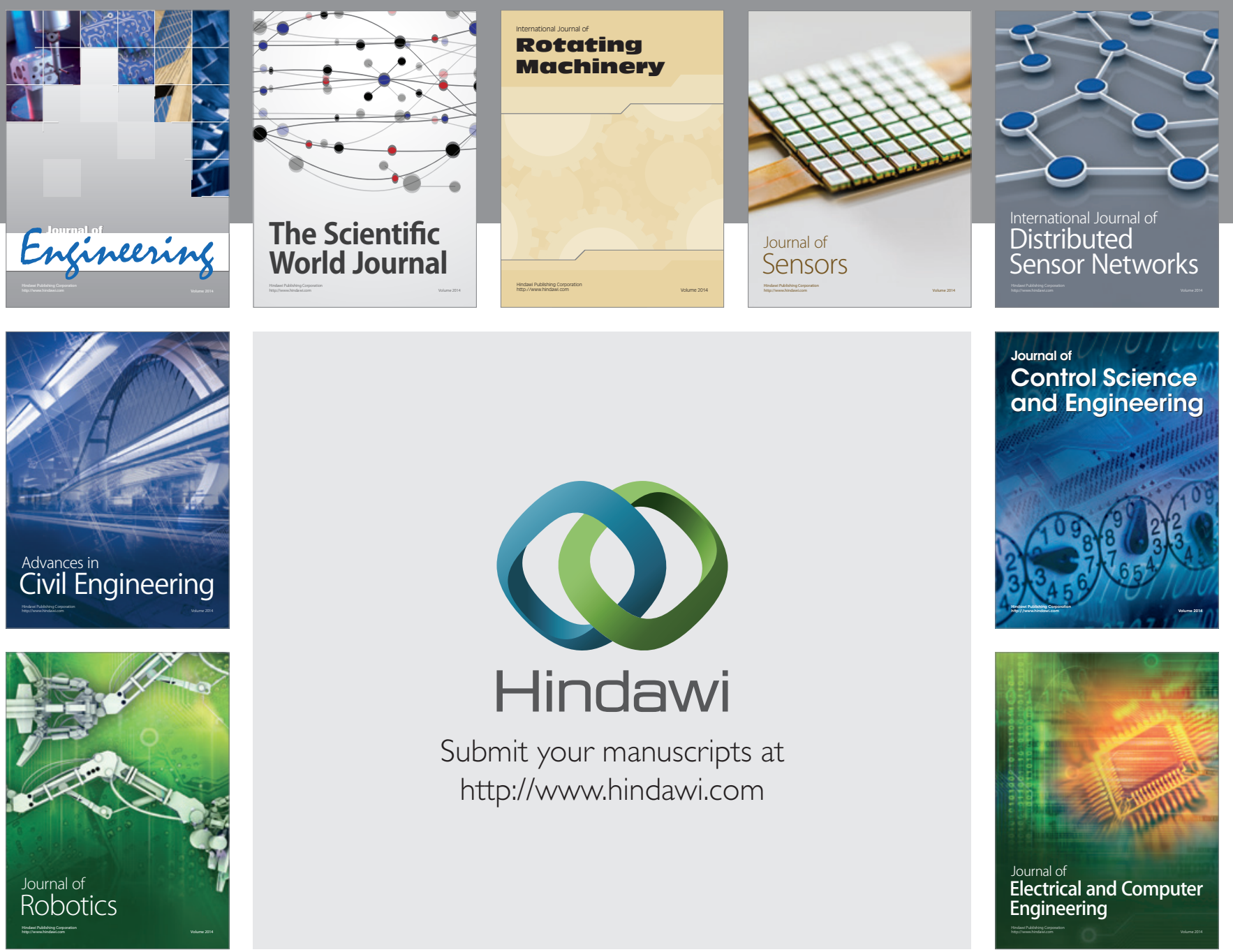

Submit your manuscripts at

http://www.hindawi.com
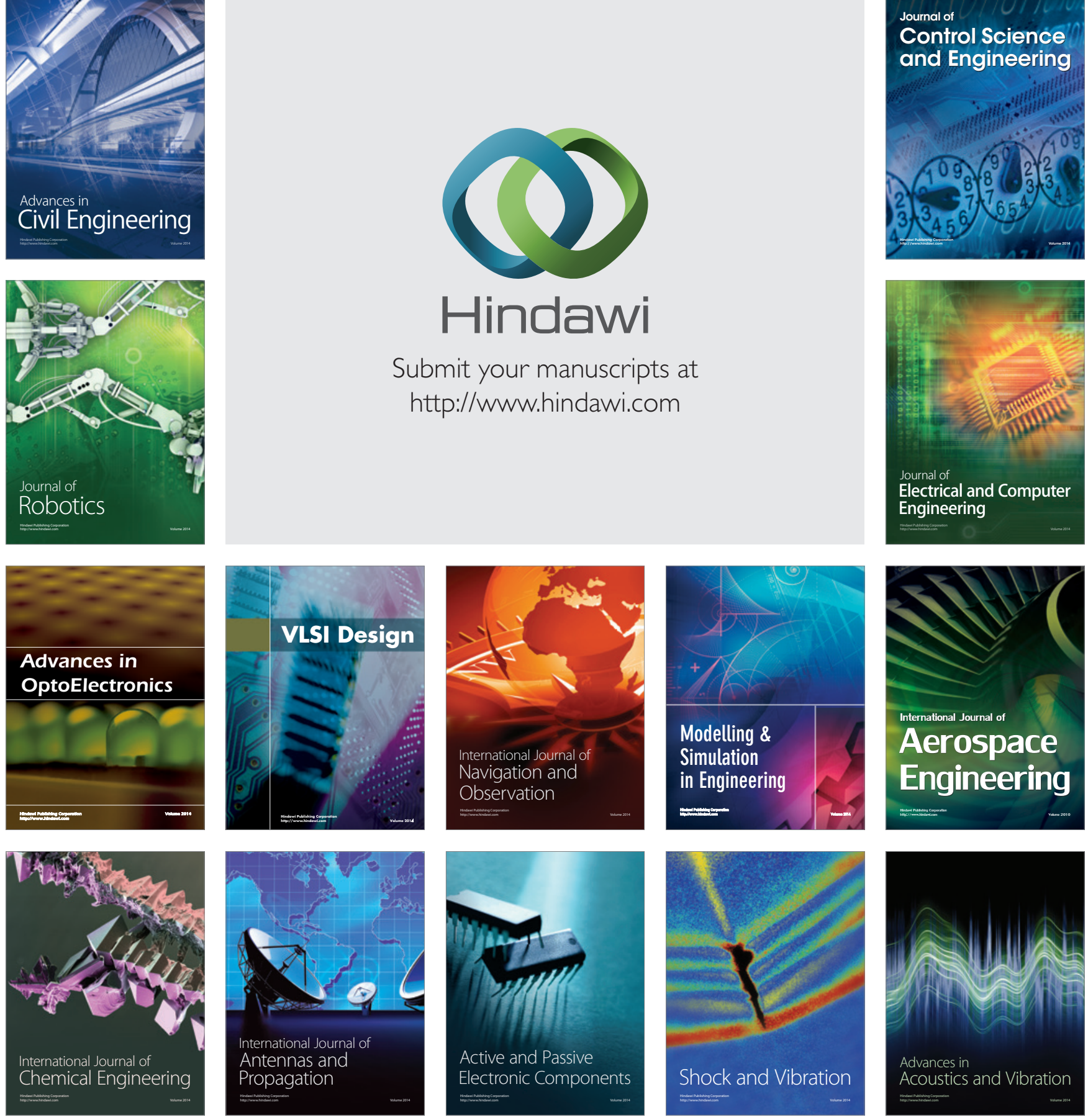